\title{
Urinary Volatile Organic Compounds as Potential Biomarkers in Idiopathic Membranous Nephropathy
}

\author{
Mingao Wang Rujuan Xie Xibei Jia Ruichan Liu \\ Department of Nephrology, The First Affiliated Hospital of Harbin Medical University, Harbin, China
}

\section{Significance of the Study}

- Six urinary volatile organic compounds were isolated from patients with idiopathic membranous nephropathy using gas chromatography/mass spectrometry. These urinary volatile organic compounds could become potential biomarkers of idiopathic membranous nephropathy.

\section{Keywords}

Urinary volatile organic compound · Gas chromatography/ mass spectrometry · Idiopathic membranous nephropathy

\begin{abstract}
Objective: To detect urinary volatile organic compounds (VOCs) in patients with idiopathic membranous nephropathy (iMN) and normal controls, and to examine whether or not urinary VOCs can act as biomarkers for the diagnosis of iMN independent of renal biopsy. Materials and Methods: Gas chromatography/mass spectrometry (GC/MS) was used to assess the urine collected from 63 iMN patients and 15 normal controls. The statistical methods of principal component analysis and partial least squares discriminant analysis were performed to process the final data in Common Data Format which were converted from GC/MS data. $R \boldsymbol{e}$ sults: Six VOCs in the urine samples of iMN patients exhibited significant differences from those of normal controls: carbamic acid monoammonium salt, 2-pentanone, 2,4-dimethyl-pentanal, hydrogen azide, thiourea, and 4-heptanone were significantly higher than in controls $(p<0.05)$.
\end{abstract}

\begin{tabular}{ll}
\hline KARGER & $\begin{array}{l}\text { ( } 2017 \text { The Author(s) } \\
\text { Published by S. Karger AG, Basel }\end{array}$ \\
$\begin{array}{l}\text { E-Mail karger@karger.com } \\
\text { www.karger.com/mpp }\end{array}$ & $\begin{array}{l}\text { This is an Open Access article licensed under the Creative Commons } \\
\text { Attribution-NonCommercial-4.0 International License (CC BY-NC) } \\
\text { (http://www.karger.com/Services/OpenAccessLicense), applicable to } \\
\text { the online version of the article only. Usage and distribution for com- } \\
\text { mercial purposes requires written permission. }\end{array}$
\end{tabular}

Conclusions: Six urinary VOCs were isolated from patients with iMN using GC/MS. The analysis of urinary VOCs using GC/MS could be developed into a non-invasive method for the detection of iMN.

\footnotetext{
(c) 2017 The Author(s)

Published by S. Karger AG, Basel
}

\section{Introduction}

Idiopathic membranous nephropathy (iMN) is a common cause of the onset nephrotic syndrome in adults [1]. Although in most patients the disease progresses relatively slowly, approximately $50 \%$ of patients with iMN if not treated will develop end stage renal disease [2]. Because of a spontaneous remission of proteinuria, iMN remains the 2 nd or 3 rd most common type of primary glomerulonephritis, resulting in end stage renal disease at a median of 5 years after diagnosis [3]. In addition, it is more likely than other causes of the nephrotic syndrome to expose the patient to the complications of venous thromboembolic events and pulmonary em- 
bolism [4], which induces high mortality from iMN. Thus, an accurate and early diagnosis is needed to treat iMN. Renal biopsy and histological evaluation remain as the standard for making a definitive diagnosis in adults, which could help to predict the clinical course and renal outcome [5]. However, renal biopsy has certain limitations: for instance, it is difficult to perform the biopsy when the patients are at high risk, such as uncontrolled bleeding diathesis, anatomic abnormalities, uncooperative behaviour, pregnancy, solitary kidney, and severe obesity [6]. Equally important, renal biopsy is expensive and inconvenient to use in primary care hospitals because it might not only cause discomfort in patients but also lead to unanticipated arteriovenous fistula and pseudoaneurysm [6, 7]. Hence, there is an urgent need to identify specific biomarkers that are simple, safe, and accurate for the early diagnosis of therapeutic intervention of iMN.

Urine has been defined as a fluid biopsy of the kidney, and pathological changes may be detected in the urinary components, which can be obtained non-invasively, in large amounts, and at minimum cost. Previous studies have suggested that urinary markers could predict the progression of iMN $[8,9]$, such as TGF- $\beta_{1}$, complement SC5b-9, $\beta_{2}$-microglobulin, and $\alpha_{1}$-microglobulin. Urine proteomics is emerging as a potential source of biomarkers that can differentiate steroid-resistant nephrotic syndrome from steroid-sensitive nephrotic syndrome. Bolignano et al. [10] reported identifying patients whose CKD progression had higher baseline urine neutrophil gelatinase-associated lipocalin levels than non-progressors. Mishra et al. [11] suggested that urinary N-acetyl- $\beta$-Dglucosaminidase activity was an extremely sensitive indicator of renal disease activity with a moderate predictive value for steroid responsiveness.

Compared with renal biopsy, the detection of specific patterns of volatile organic compounds (VOCs) in urine using gas chromatography/mass spectrometry (GC/MS) is a convenient and non-invasive technique [12]. The analysis of VOCs in patient urine using GC/MS has been shown to distinguish not just cancer from non-cancer patients but also some cancer subtypes including lung, breast, and colorectal cancer $[13,14]$. Arasaradnam et al. [15] demonstrated VOC patterns in urine that were able to distinguish coeliac disease from irritable bowel syndrome. Equally, Mochalski et al. [16] identified potential urine "markers" for the presence of entrapped persons during rescue operations, by selecting a preliminary set of VOCs spontaneously released from urine. Hence, urinary VOCs have been confirmed to be effective biomarkers in
Table 1. The characteristics of the iMN patients and normal controls

\begin{tabular}{lcc}
\hline & $\begin{array}{l}\text { iMN } \\
(n=63)\end{array}$ & $\begin{array}{l}\text { Normal controls } \\
(n=15)\end{array}$ \\
\hline $\begin{array}{l}\text { Age, years } \\
\text { Sex }\end{array} \quad 48 \pm 13$ & $50 \pm 11$ \\
$\quad$ Male & $38(60.3)$ & $9(60)$ \\
$\quad$ Female & $25(39.7)$ & $6(40)$ \\
Urinary protein, g/day & $6.4 \pm 2.7$ & $0.06 \pm 0.04$ \\
Serum albumin, g/dL & $28.6 \pm 9.5$ & $47.2 \pm 7.3$ \\
Serum creatinine, mg/dL & $1.2 \pm 0.3$ & $0.9 \pm 0.2$ \\
\hline
\end{tabular}

Data are presented as mean \pm SD or $n(\%)$. iMN, idiopathic membranous nephropathy.

many physiological and pathological states [17]. Since kidneys act as the main organs of urine metabolism, we examined whether or not urinary VOCs could replace renal biopsy for those patients who do not agree to renal biopsy.

\section{Materials and Methods}

\section{Subjects and Urine Preparation}

A total of 63 patients who were admitted to The First Affiliated Hospital of Harbin Medical University between December 2013 and November 2014 with biopsy-proven iMN were included in this study, along with 15 healthy, age-matched controls. Secondary membranous nephropathy (sMN) had been ruled out using history, imaging, serology, and routine laboratory investigations. The inclusion criteria were high levels of proteinuria: $>3.5 \mathrm{~g} /$ day, hypoalbuminemia (serum albumin): $<30 \mathrm{~g} / \mathrm{L}$, serum creatinine: $<1.5$ $\mathrm{mg} / \mathrm{dL}$, and absence of any systemic disease known to produce nephrotic syndrome. Patients were given dietary sodium restriction $(<3 \mathrm{~g} /$ day $)$ and $1 \mathrm{~g} / \mathrm{kg}$ protein intake, and none of them had received nephrotoxic drugs or radio contrast dye prior to the enrolment. Urine was collected the day before renal biopsy in standard universal Sterling specimen containers (Newport, UK) and frozen at $-80^{\circ} \mathrm{C}$ for subsequent batch analysis, within $2 \mathrm{~h}$ of collection. The Ethics Committee of Harbin Medical University approved the protocol of this study. The characteristics of the subjects are shown in Table 1.

Specimen Processing

The frozen urine samples were thawed by carefully raising the temperature of the sample to $5^{\circ} \mathrm{C}$ in a controlled procedure, and then divided into separate 5 - $\mathrm{mL}$ aliquots for analysis in each of the instruments used in this study. A sample was transferred into a 10$\mathrm{mL}$ glass vial and sealed with a crimp lid.

Solid-Phase Micro-Extraction and GC/MS Analysis

Urine samples were analysed using a Bruker Scion SQ gas chromatograph-mass spectrometer (GC-MS) fitted with a Restek Rxi-
376

Med Princ Pract 2017;26:375-380 DOI: $10.1159 / 000478782$
Wang/Xie/Jia/Liu 
Fig. 1. The 2-dimensional principal component analysis score plot between idiopathic membranous nephropathy patients and normal controls $(\mathrm{R} 2 \mathrm{X}=0.821, \mathrm{Q} 2=$ 0.549 ).

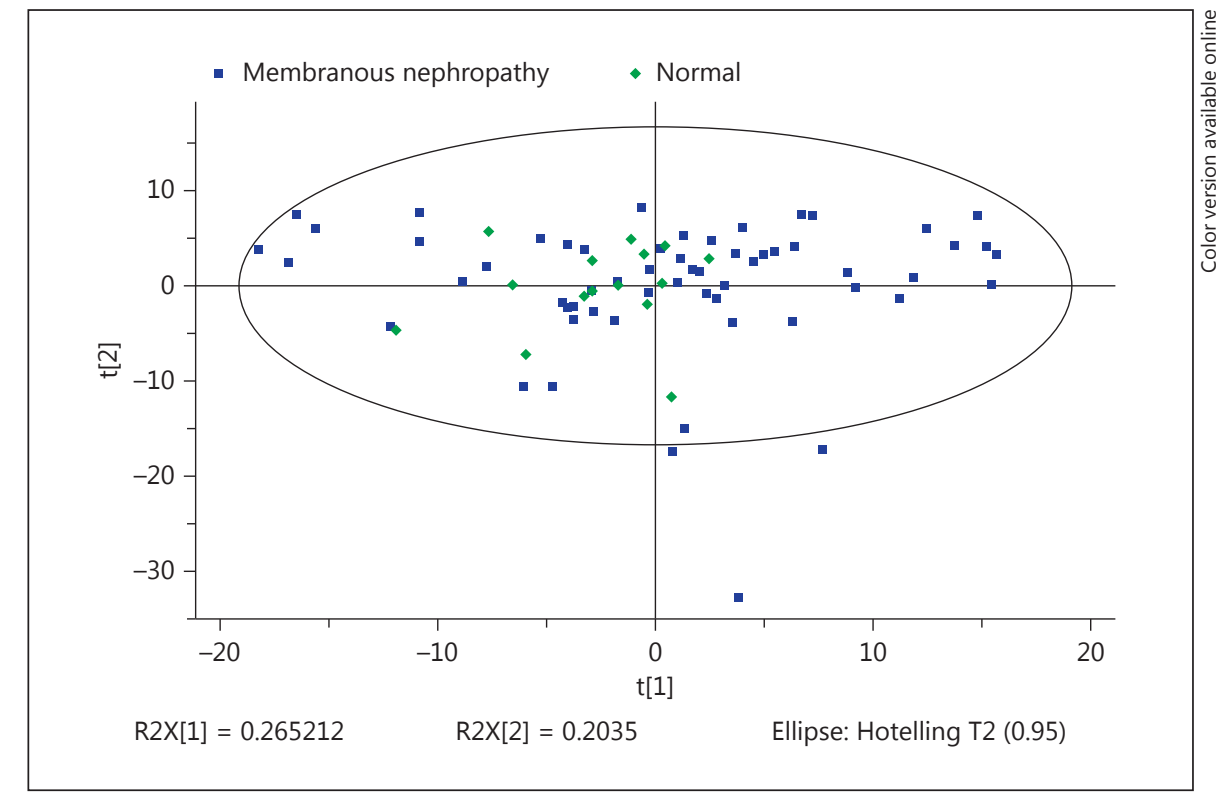

624Sil MS fused silica GC column (length $20 \mathrm{~mm}$, internal diameter $0.18 \mathrm{~mm}$, wall thickness $1.0 \mu \mathrm{m}$ ) and a CombiPAL autosampler (CTC, Switzerland). Due to the expected small concentrations of chemical components within the sample, the autosampler was improved by attaching a solid-phase micro-extraction pre-concentration fibre composed of poly-dimethylsiloxane with a thickness of $100 \mathrm{um}$. These sealed aliquots were individually heated to $60^{\circ} \mathrm{C}$ for $5 \mathrm{~min}$ before the solid-phase micro-extraction fibre was introduced into the vials for a further $10 \mathrm{~min}$ to absorb the VOCs being released into the headspace above the urine. The now-saturated fibre was then heated to $250^{\circ} \mathrm{C}$ at the GC injector port to introduce the desorbed volatiles into the machine. Samples were mixed with helium carrier gas when entering the column at a split ratio of 1:20 to maintain peak sharpness at the end detector. The GC oven followed a temperature programme for each sample in order to separate the constituent VOCs in terms of boiling point and molecular weight, by first holding at $50^{\circ} \mathrm{C}$ for $1 \mathrm{~min}$ before increasing at a constant rate of $20^{\circ} \mathrm{C} / \mathrm{s}$ up to a maximum of $280^{\circ} \mathrm{C}$. The separated compounds were detected by chromatography, and then fragmented and analysed by the mass spectrometer. Alternate 5 -mL samples of de-ionised water were run through the system in between each urine sample, in order to verify that any VOCs identified were not introduced by the external environment.

\section{Extraction and Pre-Treatment of the GC/MS Raw Data}

Raw GC/MS data were converted into Common Data Format (NetCDF) files using Shimadzu GCMS Postrun Analysis software and subsequently processed using the XCMS toolbox. The XCMS parameters consisted of the default settings with the following exceptions: $\mathrm{xcmsSet}$ ( $\mathrm{fwhm}=8$, snthresh $=6$, $\max =200$ ); retcor (method = "linear," family = "gaussian," plottype = "mdevden"), and a bandwidth of 8 for the first grouping command and 4 for the second grouping command. The data set of the aligned mass ions was exported from XCMS to Microsoft Excel for further processing to normalize the data prior to multivariate analyses.
Statistical Analyses

The normalized data were exported to SIMCA-p 11.5 for principal component analysis (PCA), partial least squares discriminant analysis (PLSDA), and orthogonal PLSDA (OPLSDA). To prevent overfitting, the default 7-round cross-validation in the SIMCA-p software was used, and permutation tests using 100 iterations was performed to further validate the supervised model. In addition, the non-parametric Kruskal-Wallis rank sum test was performed for each metabolite, and the corresponding false discovery rate based on $p$ values was calculated to correct for multiple comparisons. The potential urinary VOCs were selected as biomarkers based on variable importance in the projection values calculated from the OPLSDA model and false discovery rate values of 1.5 and 0.05 .

\section{Results}

The tight clustering of samples in the 2-dimensional PCA score plot demonstrated that the approach was effective (R2X = 0.821, Q2 = 0.549) (Fig. 1). Thus, a good separation tendency for the iMN patients and normal controls was detected from the PCA score plot. The OPLSDA score plot showed that the iMN patients were clearly separated from the normal controls using 1 predictive component and 1 orthogonal component $(\mathrm{R} 2 \mathrm{X}=$ $0.367, \mathrm{R} 2 \mathrm{Y}=0.824, \mathrm{Q} 2=0.780$ ) (Fig. 2). The supervised PLSDA model fully supported the validity of the model $(\mathrm{R} 2=[0.0,0.1], \mathrm{Q} 2=[0.0,-0.272])($ Fig. 3).

In addition, the metabolite identification performed using the National Institute of Standards and Technology database (NIST 11) showed that in iMN patients, 6 meta-

Med Princ Pract 2017;26:375-380

Urinary VOCs in iMN 
Fig. 2. The orthogonal partial least squares discriminant analysis score plot between idiopathic membranous nephropathy patients and normal controls $(\mathrm{R} 2 \mathrm{X}=0.367$, $\mathrm{R} 2 \mathrm{Y}=0.824$, Q2 $=0.780)$.

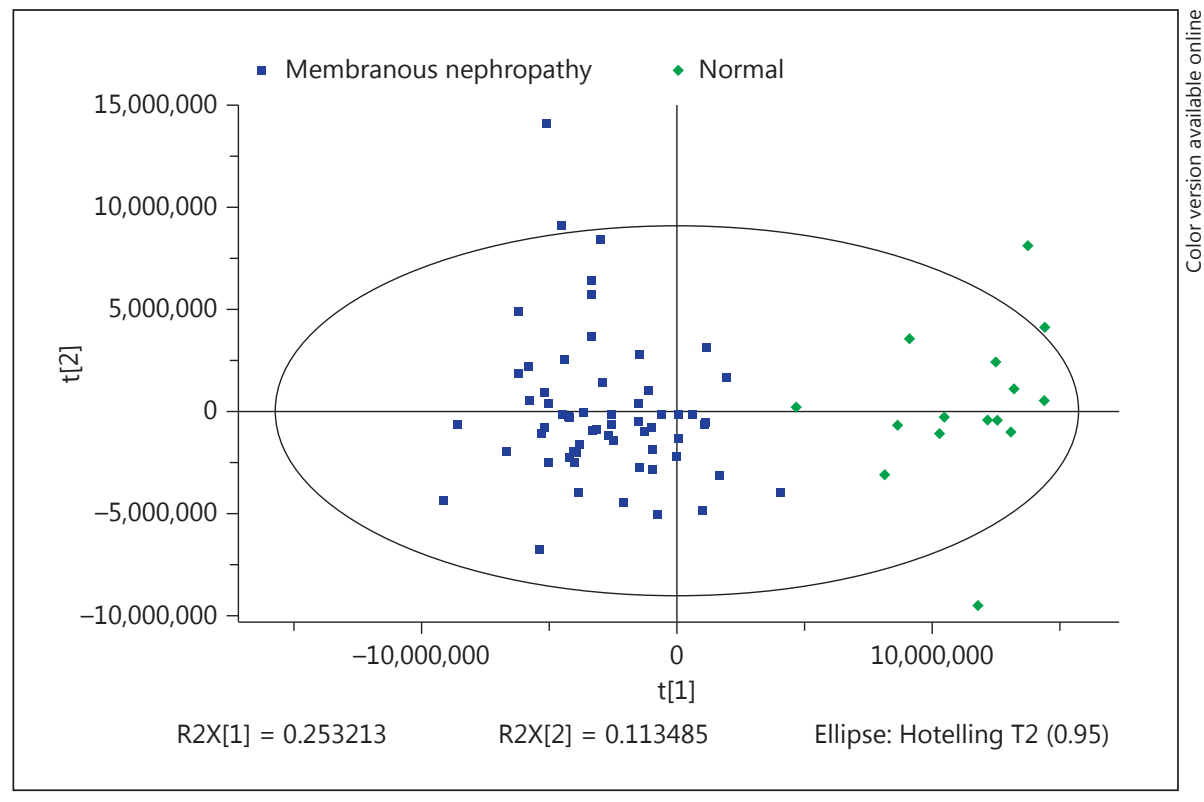

Fig. 3. Validation plot obtained from $\mathrm{Y}$ intercepts of 100 permutation tests: $\mathrm{R} 2=(0.0$, $0.1), \mathrm{Q} 2=(0.0,-0.272)$.

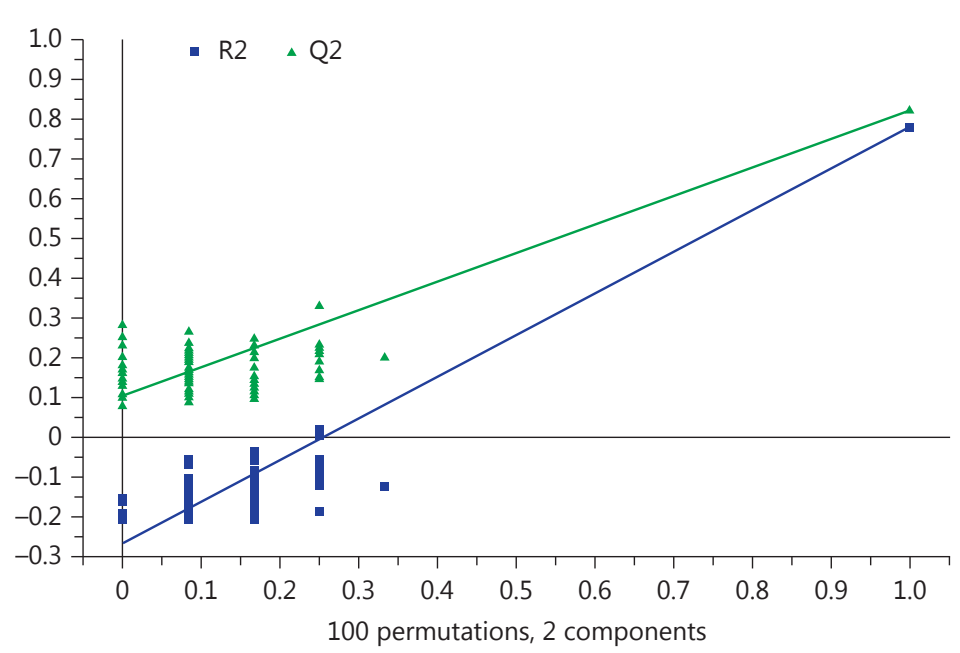

bolic VOCs were significantly greater than in the normal controls: carbamic acid monoammonium salt, 2-pentanone, 2,4-dimethyl-pentanal, hydrogen azide, thiourea, and 4-heptanone ( $p<0.05$; Table 2$)$.

\section{Discussion}

In this study, 6 urinary VOCs in iMN patients, including carbamic acid monoammonium salt, 2-pentanone, 2,4-dimethyl-pentanal, hydrogen azide, thiourea, and 4-heptanone were present at higher concentrations than those in normal controls. These 6 urinary VOCs mostly belong to ketones, aldehydes, and hydrocarbons, most of which are linked with oxidative stress and lipid metabolism [18]. Due to the kidneys' extraction of the soluble wastes from the bloodstream and pre-concentration capabilities, urine has considerable value as a promising source of volatile biomarkers. Smith et al. [19] have identified more than 200 volatiles in human urine, which belong to different chemical classes such as alcohols, ketones, hydrocarbons, pyrroles, furans, aldehydes, terpenes, sulphur-containing compounds (isocyanates, sulphides), and $O$ - and $N$-heterocyclic compounds. In re- 
Table 2. Related metabolic biomarkers in the urine of iMN patients and normal controls

\begin{tabular}{|c|c|c|c|c|}
\hline \multirow[t]{2}{*}{ Potential biomarker } & \multirow[t]{2}{*}{ RT } & \multicolumn{3}{|c|}{ iMN patients vs. normal controls } \\
\hline & & $p$ value & FC & VIP \\
\hline Carbamic acid, monoammonium salt & 68.56 & $1.33452 \mathrm{E}-06$ & 2.44177605 & 11.725 \\
\hline 2-Pentanone & 184.54 & $4.30416 \mathrm{E}-06$ & 2.998404247 & 2.93322 \\
\hline 2,4-Dimethyl-pentanal & $1,376.52$ & 0.014803966 & 1.266196027 & 2.55177 \\
\hline Hydrogen azide & 138.06 & 0.000385602 & 2.688287525 & 4.91726 \\
\hline Thiourea & 81.04 & 0.02434092 & 1.551008829 & 3.12858 \\
\hline 4-Heptanone & 328.73 & 0.005410534 & 2.152352731 & 2.81457 \\
\hline
\end{tabular}

iMN, idiopathic membranous nephropathy; RT, retention time; FC, fold change; VIP, variable importance in the projection.

cent years, urinary VOCs have received growing interest and have been an expanding area of research. In addition, numerous studies have confirmed that urinary VOCs are present in abnormal concentrations in patients diagnosed with tuberculosis [20], irritable bowel syndrome [15], and cancers such as lung, breast, and colorectal cancer $[13,14]$. In recent years, several research studies have shown that the exhaled breath VOCs could act as biomarkers of chronic kidney disease [21], while to our knowledge there is no study on the application of urinary VOCs to kidney disease territory. Hence, we focus on urinary VOCs, through which one maybe differentiate between iMN patients and normal controls.

In this study, 4-heptanone and 2-pentanone were significantly increased in iMN patients, which might indicate some relationship between oxidative stress and iMN. Since ketones were reported to be particularly abundant in human urine [22], Wahl et al. [23] have identified that 4-heptanone in urine is most probably of exogenous origin, which is supposed to be a product of $\beta$-oxidation of 2 -ethylhexanoic acid, a metabolic product of the plasticizer di-(2-ethylhexyl)-phthalate. Previous studies confirmed that 4-heptanone was related to more specific stages of diabetic polyneuropathy [24]. The source of 2-pentanone in humans has been assumed to be from increased fatty acid $\beta$-oxidation [25], and urine 2-pentanone qualitative analysis of volatile compounds has identified a biomarker for suspected inherited metabolic disorders [25].

This study demonstrated that 2,4-dimethyl-pentanal in urine VOCs was obviously higher in iMN patients compared with controls. Urine aldehydes can be both of endogenous and exogenous origin. Draper et al. [26] have reported that 2,4-dimethyl-pentanal is produced during lipid peroxidation by the so-called $\beta$-cleavage reaction of lipid alkoxyl radicals. It is widely known that there is lip-

Urinary VOCs in iMN id metabolic disturbance in patients with nephrotic syndrome, so the result may be explained by the lipid peroxidation damage that occurs in iMN patients.

In this study, urine thiourea VOCs increased significantly in iMN patients, who neither had thyroid disease nor took thiourea analogue drugs orally. Thiourea, which is structurally very similar to urea, is a powerful scavenger of hydroxyl radicals [27]. Urinary thiourea concentration is considered as a well-established biomarker for the assessment of thyroid disease [28]. Fustinoni et al. [29] have identified the determination of urinary ethylenethiourea in humans by GC/MS. However, there is no report on urinary thiourea VOCs, and further study is needed to find out the necessity and importance of urine thiourea VOCs. In our study, hydrogen azide and carbamic acid (monoammonium salt of urinary VOCs) were both significantly increased in iMN patients compared with controls. It has been reported that carbamic acid monoammonium salt is an important organic raw material of rubber auxiliary material, binder, UV absorbent, dyes, pharmaceuticals, explosives, etc. [30]. However, previous studies showed no data on the 2 urinary VOCs. The mechanism for the generation of urinary VOCs is one of the main subjects of current research, but they are still perturbed in many physiological and pathological states, which include health status, diet habit, physical stress, environmental exposure, and disease states. The origin of urinary VOCs in many cases is still unclear and demands more research.

In the present study, 4-heptanone, 2-pentanone, and 2,4-dimethyl-pentanal have the potential to act as markers for iMN patients, which leads to the possibility that the analysis of urinary VOCs could be further developed into a convenient and non-invasive method for the detection of iMN. Urine sample compared with renal biopsy is suit-

Med Princ Pract 2017;26:375-380 DOI: $10.1159 / 000478782$ 
able as a convenient source for screening kidney diseases. The biological significance of the urinary VOCs identified as biomarkers for iMN needs more exploration. In addition, this study did not distinguish among patients with different stages of iMN. Thus, future studies involving larger samples, examinations of different pathological types of nephrotic syndrome, and detailed classification of iMN are required to pursue these possibilities.

\section{Conclusion}

Six urinary VOCs were isolated from patients with iMN using GC/MS. Compared with normal controls, iMN had unique urinary VOC profiles. These urinary VOCs could be potential biomarkers in iMN. The analysis of urinary VOCs using GC/MS might develop into a non-invasive detection method of iMN.

\section{References}

1 Liu H, Luo W, Gong S, et al: Detection and clinical significance of glomerular M-type phospholipase A2 receptor in patients with idiopathic membranous nephropathy. Intern Med J 2016;46:1318-1322.

2 du Buf-Vereijken PW, Branten AJ, Wetzels JF: Idiopathic membranous nephropathy: Outline and rationale of a treatment strategy. Am J Kidney Dis 2005;46:1012-1029.

3 Maisonneuve P, Agodoa L, Gellert R, et al: Distribution of primary renal diseases leading to end-stage renal failure in the United States, Europe, and Australia/New Zealand: results from an international comparative study. Am J Kidney Dis 2000;35:157-165.

4 Kerlin BA, Ayoob R, Smoyer WE: Epidemiology and pathophysiology of nephrotic syndrome associated thromboembolic disease. Clin J Am Soc Nephrol 2012;7:513-520.

5 Hofstra JM, Wetzels JF: Management of patients with membranous nephropathy. Nephrol Dial Transplant 2012;27:6-9.

6 Eiro M, Katoh T, Watanabe T: Risk factors for bleeding complications in percutaneous renal biopsy. Clin Exp Nephrol 2005;9:40-45.

7 Simard-Meilleur M-C, Troyanov S, Roy L, et al: Risk factors and timing of native kidney biopsy complications. Nephron Extra 2014;4: $42-49$.

8 van den Brand JA, Hofstra JM, Wetzels JF: Prognostic value of risk score and urinary markers in idiopathic membranous nephropathy. Clin J Am Soc Nephrol 2012;7:12421248.

9 Kon SP, Coupes B, Short CD, et al: Urinary C5b-9 excretion and clinical course in idiopathic human membranous nephropathy. Kidney Int 2014;48:1953-1958.

10 Bolignano D, Lacquaniti A, Coppolino G, et al: Neutrophil gelatinase-associated lipocalin (NGAL) and progression of chronic kidney disease. Clin J Am Soc Nephrol 2009;4:337344

11 Mishra OP, Jain P, Srivastava P, et al: Urinary $\mathrm{N}$-acetyl-beta-D glucosaminidase (NAG) level in idiopathic nephrotic syndrome. Pediatr Nephrol 2012;27:589-596.
12 Khalid T, White P, De Lacy Costello B, et al: A pilot study combining a GC-sensor device with a statistical model for the identification of bladder cancer from urine headspace. PLoS One 2013;8:e69602.

13 Cornu J-N, Cancel-Tassin G, Ondet V, et al: Olfactory detection of prostate cancer by dogs sniffing urine: a step forward in early diagnosis. Eur Urol 2011;59:197-201.

14 Sonoda H, Kohnoe S, Yamazato T, Satoh Y, et al: Colorectal cancer screening with odour material by canine scent detection. Gut 2011; 60:814-819.

15 Arasaradnam RP, Westenbrink E, McFarlane $\mathrm{M}$, et al: Differentiating coeliac disease from irritable bowel syndrome by urinary volatile organic compound analysis - a pilot study. PLoS One 2014;9:e107312.

16 Mochalski P, Krapf K, Ager C, et al: Temporal profiling of human urine VOCs and its potential role under the ruins of collapsed buildings. Toxicol Mech Methods 2012;22:502511.

17 Arasaradnam RP, McFarlane M, Daulton E, et al: Non-invasive distinction of non-alcoholic fatty liver disease using urinary volatile organic compound analysis: early results. J Gastrointestin Liver Dis 2015;24:197-201.

18 Hakim M, Broza YY, Barash O, et al: Volatile organic compounds of lung cancer and possible biochemical pathways. Chem Rev 2012; 112:5949-5966.

19 Smith S, Burden H, Persad R, et al: A comparative study of the analysis of human urine headspace using gas chromatography-mass spectrometry. J Breath Res 2008;2:037022.

20 Banday KM, Pasikanti KK, Chan EC, et al: Use of urine volatile organic compounds to discriminate tuberculosis patients from healthy subjects. Anal Chem 2011;83:55265534 .
21 Mochalski P, King J, Haas M, et al: Blood and breath profiles of volatile organic compounds in patients with end-stage renal disease. BMC Nephrol 2014;8;15-23.

22 Mochalski P, Krapf K, Ager C, et al: Temporal profiling of human urine VOCs and its potential role under the ruins of collapsed buildings. Toxicol Mech Methods 2012;22:502511.

23 Wahl HG, Hong Q, Hildenbrand S, et al: 4-Heptanone is a metabolite of the plasticizer di(2-ethylhexyl) phthalate (DEHP) in haemodialysis patients. Nephrol Dial Transplant 2004; 19:2576-2583.

24 Mills GA, Walker V: Headspace solid-phase microextraction profiling of volatile compounds in urine: application to metabolic investigations. J Chromatogr B Biomed Sci Appl 2001;753:259-268.

25 Yancey M, Holland ML, Stuart R, et al: Urinary profiles of organic acids and volatile metabolites during the starvation process in rats. J Chromatogr B Biomed Sci Appl 1986;382: 3-18.

26 Draper HH, Csallany AS, Hadley M: Urinary aldehydes as indicators of lipid peroxidation in vivo. Free Radic Biol Med 2000;29:10711077.

27 Bellinger DC: Lead neurotoxicity and socioeconomic status: conceptual and analytical issues. Neurotoxicology 2008;29:828-832.

28 Axelstad M, Boberg J, Nellemann C, et al: Exposure to the widely used fungicide mancozeb causes thyroid hormone disruption in rat dams but no behavioral effects in the offspring. Toxicol Sci 2011;120:439-446.

29 Fustinoni S, Campo L, Colosio C, et al: Application of gas chromatography-mass spectrometry for the determination of urinary ethylenethiourea in humans. J Chromatogr B Analyt Technol Biomed Life Sci 2005;814: 251-258.

30 Rayat S, Qian M, Glaser R: Nitrosative cytosine deamination. An exploration of the chemistry emanating from deamination with pyrimidine ring-opening. Chem Res Toxicol 2005;18:1211-1218. 